\title{
Griginal Gastrointestinal spectrum of dengue fever in a dengue epidemic
}

\author{
Indrajeet Kumar Tiwary ${ }^{1}$, Avijit Das ${ }^{2}$, Mahesh Kumar \\ Goenka ${ }^{3}$
}

ABSTRACT

${ }^{I}$ Consultant Gastrointestinal intensivist, Institute of Gastrosciences, Apollo

Gleneagles Hospital Kolkata.

${ }^{2}$ Registrar, Institute of Gastrosciences, Apollo Gleneagles Hospital Kolkata.

${ }^{3}$ Director and Head, Institute of

Gastrosciences, Apollo Gleneagles

Hospitals, Kolkata.

Correspondence: Indrajeet Kumar Tiwary

Email: tiwaryindrajeet@yahoo.co.in

Background: Every year thousands of individuals are affected by dengue viral infections in India. It has been a major public health problem in our country, in view of fatal outbreaks in the last few decades. In addition to classical clinical features, dengue may manifest with various atypical complications.

Methods: We did a cross-sectional study on 35 patients in our gastroenterology department during the dengue epidemic which occurred in 2013-2014.

Results: During this period a total of 219 patients were admitted in our hospital. The objective was to study the gastrointestinal (GI) Spectrum of dengue fever in a tertiary care centre with special focus on atypical presentation. We found $15.9 \%$ (35 out of 219 patients) of the patients diagnosed as dengue with GI manifestations such as cholecystitis, hepatitis, appendicitis and pancreatitis.

Conclusion: Dengue fever presents with a myriad of gastrointestinal manifestations which include hepatitis, cholecystitis, pancreatits and appendicitis.

KEYWORDS: India, dengue, cholecystitis, hepatitis, appendicitis, pancreatitis.

\section{Introduction}

Dengue is the most rapidly spreading mosquitoborne viral disease in the world. All dengue serotypes (DENV1-DENV4) circulate in most countries of the Asian continent. Dengue fever is common in tropical countries including India with frequent outbreaks in the rainy season. Over 2.5 billion people, i.e. $40 \%$ of the world's population, are now at risk from dengue. WHO currently estimates there may be 50-100 million dengue infections worldwide every year. ${ }^{1}$ Dengue may manifest either as a mild self-limitingdisease or may lead to severe complications including dengue hemorrhagic fever (DHF) and dengue shock syndrome (DSS). ${ }^{2,3}$ Few cases 
of uncommon complications such as acute myocarditis, acute hepatic failure, acalculous cholecystitis, and acute pancreatitis have been reported. ${ }^{4,5}$ In this study, we have attempted to point out mainly the various typical and atypical features of dengue related to the gastrointestinal tract, its complications, and the changes in laboratory parameters in dengue fever as seen during this dengue epidemic which occurred in our region.

\section{Methods}

This is a descriptive, cross sectional study done in the Department of Gastroenterology at our hospital, between the months of September 2013 to February 2014. At our hospital 219 patients were diagnosed to have dengue of which 35 patients (15.9\%) had gastrointestinal manifestations.

All the cases of acute febrile illness with various typical and atypical clinical features, admitted at the Department of Gastroenterology of our hospital who were serologically diagnosed as Dengue fever as per the WHO criteria, were included in the study. WHO criteria $^{6}$ for diagnosis of Dengue fever includes- Isolation of the dengue virus from serum or autopsy samples or demonstration of a fourfold or greater increase in reciprocal $\operatorname{IgG}$ or $\operatorname{IgM}$ antibody titers to one or more dengue virus antigens in paired serum samples or demonstration of dengue virus antigen in autopsy tissue, serum or cerebrospinal fluid samples by immunohistochemistry, immunofluorescence or ELISA ( Enzyme-linked immunosorbent assay) or detection of dengue virus genomic sequences in autopsy tissue, serum or CSF samples by polymerase chain reaction.

Proper clinical history was taken and physical examination was done in all cases. Investigations done besides dengue serology (dengue NS1, IgM, IgG) included serial hemograms, renal function tests (RFT), liver function tests (LFT), urinalysis and chest roentgenogram. Ultrasound abdomen, prothrombin time (PT), INR and activated partial thromboplastin time (aPTT) were done when indicated. Infections by hepatotropic viruses, malaria, leptospira and typhoid were excluded by relevant tests as required.

\section{Results}

A total of 35 patients admitted during the study period tested positive for IgM antibody titers to one or more dengue virus antigens in paired serum samples as per protocols standardized in the laboratory. Male:female ratio was 19:16 and the mean age was 41.6 years. Among the 35 patients who tested positive for IgM antibody, 25 (71.4\%) were dengue NS1 antigen positive and 24 (68.5\%) were positive for both IgG and IgM dengue antibody.

Table 1 outlines the various clinical manifestations of these 35 patients. A total of $31(88.5 \%)$ patients had significant complications while $4(11.4 \%)$ had only pain abdomen but no significant complications. Those with complications included 9 (25.7\%) with ascites, 5 (14.3\%) with gastrointestinal bleeding, 3 (8.6\%) with acute cholecystitis (USG excluded gallstone), 3 (8.6\%) with acute viral hepatitis, $2(5.7 \%)$ with acute appendicitis, $2(5.7 \%)$ with acute pancreatitis. Out of the remaining 7 patients who were admitted with non specific pain abdomen, we found renal impairment in 2 (5.7\%), haemophagocytic syndrome in $2(5.7 \%)$, seizure disorder in $1(2.8 \%)$, intracranial bleeding in $1(2.8 \%)$ and multiple organ system failure in $1(2.8 \%)$.

While evaluating the liver function parameters in our study population we found the number of patients with total bilirubin $(\mathrm{mg} / \mathrm{dl})$ of $<2$ was $27(77.1 \%)$, in the range of $2-5$ was $5(14.3 \%)$ and $>5$ was $3(8.6 \%)$. Distribution of patients according to elevations of liver enzymes, aspartate transaminase and alanine transaminase (AST and ALT) are given in Table 2. None of them had platelets count of $<20,000 /$ cu.mm (Figure 1). Number of

Table 1: Distribution of dengue patients according to complications

\begin{tabular}{|c|l|c|c|}
\hline Srno & Complications & No of patients & Percentage \\
\hline 1 & Ascites & 9 & 25.71 \\
\hline 2 & Gastrointestinal bleeding & 5 & 14.28 \\
\hline 3 & Acute cholecystitis & 3 & 8.57 \\
\hline 4 & Acute viral hepatitis & 3 & 8.57 \\
\hline 5 & Acute appendicitis & 2 & 5.71 \\
\hline 6 & Acute pancreatitis & 2 & 5.71 \\
\hline 7 & Non specific pain abdomen with renal impairment & 2 & 5.71 \\
\hline 8 & Non specific pain abdomen with haemophagocytic syndrome & 2 & 5.71 \\
\hline 9 & Non specific pain abdomen with seizure disorder & 1 & 2.58 \\
\hline 10 & Non specific pain abdomen with intracranial bleed & 1 & 2.58 \\
\hline 11 & Non specific pain abdomen with multiple organ system failure & 1 & 2.58 \\
\hline 12 & No significant complications & 4 & 11.42 \\
\hline & Total & 35 & 100 \\
\hline
\end{tabular}


patients with INR $<1$ was $22(62.8 \% ; n=35)$ and INR in the range of $1-2$ was $13(37.1 \% ; n=35) 18(51.4 \%)$ out of 35 had creatinine $<1 \mathrm{mg} / \mathrm{dl}$ and $17(48.6 \%)$ out of $35 \mathrm{had}$ creatinine in the range of $1-3 \mathrm{mg} / \mathrm{dl}$.

While evaluating duration of hospital stay, we found that majority of the patients (i.e. 17 out of 35, 48.6\%) stayed for 5-10 days (Figure 2). Maximum mean hospital stay of 9.83 days was seen in the 20-30 years age group and lowest being of 2 days in the age group of $>20$ years.

Out of $35.5(14.3 \%)$ required major support (ventilation:4, dialysis:1) and 1 (2.8\%) succumbed to multiple organ system failure. Co-existing disease included $6(17.1 \% ; \mathrm{n}=35)$ with type 2 diabetes mellitus, 3 $(8.6 \% ; n=35)$ with hypertension and $3(8.6 \% ; n=35)$ with hypothyroidism. $7(20 \%$; $\mathrm{n}=35)$ developed sepsis and required antibiotics.

\section{Discussion}

Dengue fever has emerged as a serious international public health threat with almost half of the world's population at risk of infection. ${ }^{7}$ Although greater than 50 million cases of dengue fever are estimated to occur each year ${ }^{8}$, a large proportion of infections are asymptomatic. ${ }^{9}$ Why infection progresses to clinical disease in some persons, and not in others, is not clear? Dengue fever usually presents as an acute febrile illness, musculoskeletal pain, nausea, vomiting, and petechial rash. It may present as acute abdomen leading to diagnostic dilemma.

In the present study, we noted ascites, GI bleed, acute cholecystitis, acute hepatitis, pancreatitis and appendicitis as major gastrointestinal complications of dengue. These manifestations in patients with dengue have been reported earlier as isolated cases or case series. ${ }^{10-19}$ Ahmad et al. ${ }^{13}$ in a study on dengue patients from Pakistan, reported acute cholecystitis in 5.26\%, pancreatitis in $2.17 \%$, GI bleed in $2.73 \%$ and appendicitis in $0.46 \%$ patients. Goyal et al. ${ }^{14}$ in a study from North India, reported hepatitis in $45 \%$, pancreatitis in $7 \%$ and serositis in $6 \%$ patients with dengue fever. Khor et al. reviewed 328 patients with DHF/DSS in a dengue epidemic area in southern Taiwan. They found that out of 328 patients with DHF/DSS only 14 had acute abdomen.
Table 2: Distribution of dengue patients according to elevations of liver enzymes (AST and ALT)

\begin{tabular}{|l|c|c|}
\hline Range of AST and ALT (U/L) & No of patients (for AST); $n=35$ & No of patients (for ALT); $n=35$ \\
\hline$<40$ & $2(5.71 \%)$ & $4(11.42 \%)$ \\
\hline $40-100$ & $9(25.71 \%)$ & $15(42.86 \%)$ \\
\hline$>100$ & $24(68.57 \%)$ & $16(45.71 \%)$ \\
\hline
\end{tabular}

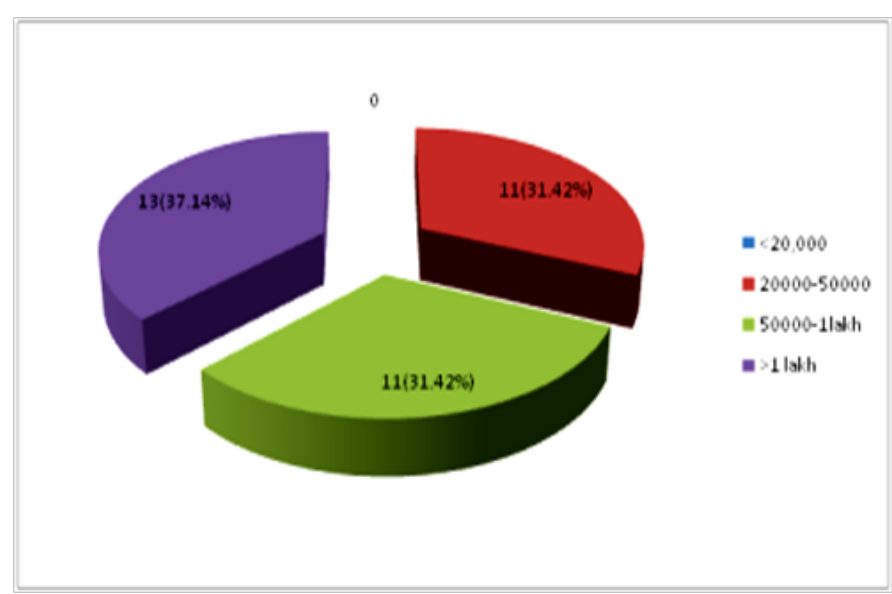

Figure 1: Distribution of the dengue patients on the basis of platelet counts.

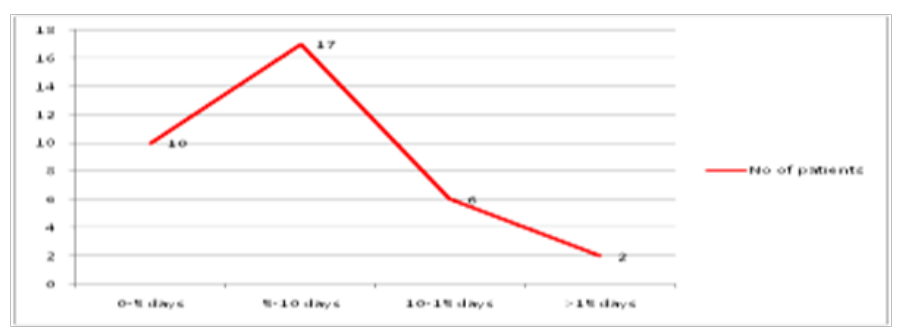

Figure 2: Distribution of number of dengue patients according to duration of hospital stay (in days).

Causes of acute abdomen were acute cholecystitis (6 acalculous and 4 calculous cholecystitis) in 10 patients, nonspecificperitonitis in 3 patients, and acute appendicitis in only 1 patient. ${ }^{5}$ Shamim reviewed 357 patients with dengue fever and reported that 276 patients had nonspecific abdominal pain without overt abdominal signs, 43 (12.04\%) had acute abdominal pain with definite abdominal signs, and only 38 (10.64\%) presented without abdominal pain. ${ }^{15}$ Premaratna et al. reported 12 cases of dengue fever mimicking acute appendicitis. ${ }^{16}$ Higher percentage of these complication in our study reflects a bias due to evaluation in patients admitted at a tertiary center in its gastroenterology department. 
Data is scarce on liver involvement in adult patients with dengue virus infection. In our study we found $8(22.8 \%)$ patients had bilirubin $>2 \mathrm{mg} / \mathrm{dl}, 24$ (68.6\%) had AST $>100 \mathrm{U} / \mathrm{L}$ and 16 (45.71\%) had ALT $>100 \mathrm{U} / \mathrm{L}$. A study done by Shukla et al. ${ }^{20}$ evaluating hepatic dysfunction in dengue showed $100 \%$ patients had an elevated AST level while 91\% had elevated ALT level and in patients who had raised levels of both enzymes, the AST levels were 2-3 times higher than ALT levels.

Kuo et $\mathrm{a}^{21}$ reported $90 \%$ of the patients in their study had abnormal AST levels, while abnormal levels of ALT and bilirubin were found in $80 \%$ and $7 \%$ respectively. Liver involvement occurs through an inflammatory process in the parenchyma provoked directly or indirectly by the virus, reducing the diameter of the lumen of the biliary canaliculus, causing obstruction and leading to bilirubinemia or even jaundice. Cholestasis with gallbladder distension and cystic duct spasm have been suggested as possible causes of acalculous cholecystitis in dengue fever.

Although 6 (17.1\%) out of 35 patients had bleeding manifestations (5 GI bleed and 1 intracranial bleed) none of the patients in our study group had platelets $<20,000 / \mu 1$ and $13(37.1 \%)$ patients had platelets $>1$ lakh. 6 patients had leucocyte count $<4000 / \mu 1,3$ patients had leucocyte count $>12000 / \mu 1$ and rest i.e 26 patients had leucocyte count in the range 4000-12000/ $\mu$ l. Although thrombocytopenia and leukopenia are not diagnostic of dengue fever, they can contribute towards suspecting a diagnosis of dengue in a patient presenting with acute abdomen, during a dengue epidemic. ${ }^{22}$ Usually patients presenting with acute appendicitis, cholecystitis and pancreatitis have a higher leukocyte count but a normal leukocyte count certainly does not rule out these conditions. Therefore patients presenting with clinical features suggestive of acute appendicitis and a normal leukocyte count need a careful approach in a dengue epidemic area. ${ }^{16}$

Primary infection with any dengue serotype induces an immune response that protects against later infection by that serotype; however, subsequent infection by another serotype, termed secondary dengue infection, is a risk factor for dengue hemorrhagic fever, which is associated with significant morbidity and occasionally death. ${ }^{23-25}$ In our study we found $24(68.6 \%$; $=35)$ patients were positive for both $\operatorname{IgG}$ and $\operatorname{IgM}$ dengue antibody i.e. having probable secondary dengue viral infection, though $\mathrm{IgG}$ avidity testing was not done.

\section{Conclusion}

Our study shows that the prevalence of dengue during this epidemic was slightly more among males compared to females and mostly affected the middle age group rather than extremes of age. Secondary viral infections were likely to have more complications compared to primary infections. Though ascites and GI bleed may have been the most common manifestations of dengue fever, atypical associations of dengue fever with surgical emergencies such as acute acalculous cholecystitis, appendicitis and pancreatitis should be kept in mind and one should be aware of this overlapping presentation in order to prevent unnecessary surgery-related morbidity or even mortality. Liver enzymes profile (usually AST $>$ ALT) as reported in this study during this epidemic should be noted for liver involvement while dealing with a suspected case of dengue fever. Bleeding manifestation in a dengue virus infected patient was not always associated with low platelet count and should therefore be observed for even in patients with a normal or slightly low platelet count along with monitoring of other coagulation parameters.

\section{References}

1. Bhatt S, Gething PW, Brady OJ, et al. The global distribution and burden of dengue. Nature. 2013;496:504507.

2. Khanna S, Vij JC, Kumar A, Singal D, Tandon R. Dengue fever is a differential diagnosis in patients with fever and abdominal pain in an endemic area. Ann Trop Med Parasitol. 2004;98:757-760.

3. Kyle JL, Harris E. Global spread and persistence of dengue. Ann Rev Microbiol. 2008;62:71-92.

4. McFarlane MEC, Plummer JM, Leake PA et al.,Dengue fever mimicking acute appendicitis: a case report. Int $J$ Surg Case Rep. 2013;4:1032-1034.

5. Khor BS, Liu JW, Lee IK, Yang KD. Dengue hemorrhagic fever patients with acute abdomen: clinical experience of 14 cases. Am J Trop Med Hyg. 2006;74:901-904.

6. Bandyopadhyay S, Lum LCS, and Kroegar A. Classifying 
dengue: a review of difficulties in using the WHO case classification of dengue haemorrhagic fever. Trop Med Int Health. 2006;11:1238-1255.

7. Centers for Disease Control and Prevention CDC Dengue Fever home page. 2005. [cited 2007 Mar 21]. Available from http://www.cdc.gov/ncidod/dvbid/dengue/index.htm

8. World Health Organization Fact sheet no. 117, dengue and dengue haemorrhagic fever. Geneva: The Organization; 2002

9. Balmaseda A, Hammond SN, Tellez Y, Imhoff L, Rodriguez Y, Saborio SI, et al. High seroprevalence of antibodies against dengue virus in a prospective study of schoolchildren in Managua, Nicaragua. Trop Med Int Health. 2006;11:935-942.

10. Thulkar S, Sharma S, Srivastava DN, Sharma SK, Berry M,Pandey RM. Sonographic findings in grade III dengue hemorrhagic fever in adults. $J$ Clin Ultrasound. 2000;28:34-37.

11. Wu KL, Changchien CS, Kuo CM. Dengue fever with acute acalculous cholecystitis. Am J Trop Med Hyg. 2003;68:657-660.

12. Lawn SD, Tilley R, Lloyd G, Finlayson C, Tolley H, Newman $P$, et al. Dengue hemorrhagic fever with fulminant hepatic failure in an immigrant returning to Bangladesh. Clin Infect Dis. 2003;37:1-4.

13. Farooq Ahmad, Ahmad Nadeem, Muhammad Saad Faisal, Mubasher Shaukat, Kashif Siddique Management Experience of Surgical Complications of Dengue Fever Patients at Hameed Latif Hospital, Lahore. Annals. 2013;19:49-54.

14. Goyal V, Gill GS, Singh J, et al. Clinical spectrums of dengue fever in a tertiary care centre with particular references to atypical presentation in the 2011 outbreak at Bathinda, Punjab, India. Int J Pharmacy Pharma Sci.
2013;5:363-367.

15. Shamim M. Frequency, pattern and management of acute abdomen in dengue fever in Karachi, Pakistan. Asian $J$ Surg. 33;2010:107-113.

16. Premaratna R, Bailey MS, Ratnasena BGN, de Silva HJ. Dengue fever mimicking acute appendicitis. Trans $R$ Soc Trop Med Hyg. 2007;101:683-685.

17. Mourão MP, De Lacerda MV, Bastos M, Albuquerque BC, and Alecrim WD. Dengue hemorrhagic fever and acute hepatitis: A case report. Braz J Infect Dis. 2004;8:461-464.

18. Wu KL, Changchien CS, Moukuo C,Chuah Sk. Dengue fever with acute acalculous. Am J Trop Med Hyg. 2003;68:657-660.

19. Kang J, Yoon SY. Dengue fever mimicking acute appendicitis: A case report. $J$ Infect Chemother. 1993;41:793-802.

20. Shukla V, Chandra A.A Study of Hepatic Dysfunction in Dengue. JAPI. 2013;61:460-461.

21. Kuo CH, Tai DI, Chang-Chien CS, et al. Liver biochemical tests and dengue fever. Am J Trop Med Hyg. 1992;47:265270.

22. Kumar L, Singh M, Saxena A, et al. Unusual Presentation of Dengue Fever Leading to Unnecessary Appendectomy. Trans R Soc Trop Med Hyg. 2015;2015:1-2.

23. Morens D.M., Fauci A.S. Dengue and hemorrhagic fever. A potential threat to public health in the United States. JAMA. 2008;299:214-216.

24. Rothman AL, Ennis FA. Immunopathogenesis of dengue hemorrhagic fever. Virology. 1999;257:1-6.

25. Vaughn DW, Nisalak A, Solomon T, et al. Rapid serologic diagnosis of dengue virus infection using a commercial capture ELISA that distinguishes primary and secondary infections. Am. J. Trop. Med. Hyg. 1999;60:693-698. 